\title{
Desensitized motion planning for underactuated multibody systems
}

\author{
Paolo Boscariol, Dario Richiedei
}

\author{
Dipartimento di Tecnica e Gestione dei Sistemi Industriali \\ Università degli Studi di Padova \\ Stradella S. Nicola 3, 36100 Vicenza, Italy \\ paolo.boscariol@unipd.it \\ dario.richiedei@unipd.it
}

\section{FULL PAPER}

\section{Abstract}

Model-plant mismatches can severely limit the effectiveness of conventional model-based motion design methods. To solve this issue, a method for robust trajectory planning that can reduce the effects of parametric uncertainties is presented in this work. The method is based on an indirect variational formulation, which is translated into a Two-Point Boundary Value Problem (TPBVP) and then solved numerically. Robustness is obtained by incorporating into the problem the sensitivity functions of the plant, and imposing some additional constraints on the initial and final points of the trajectory. A formulation aimed at reducing both the residual and the transient oscillations, as well as keeping small the control effort, is also proposed. The work presents a numerical verification of the effectiveness of the method for an underactuated system, such as a double-pendulum crane, by showing its effectiveness and robustness when performing fast rest-to-rest motions.

\section{Introduction}

High-speed and precise motion of underactuated multibody systems is challenging since this kind of mechatronic systems requires suitable trajectory profiles for limiting the vibrations that can incur both during the motion and after motion completion. The problem has been dealt with in countless works, facing it either as a control design problem or as a motion design problem. The latter approach might be convenient in all applications in which the use of a sophisticated control technique is limited by the unavailability of high-bandwidth sensors [1]. A common classification of the vast literature on motion design marks the distinction between model-free and model-based approaches. The first ones are mainly based on the use of properly interpolated time laws to define either the motion in the joint space or in the operational space. Vibration reduction and precise tracking are enforced by minimizing kinematic quantities, such as the jerk, or mixed performance indexes, thus enhancing the smoothness of the motion profile $[2,3]$. Such methods are often of convenient application due to their rather simple implementation and for being suitable to a wide range of applications.

Model-based planning methods, in contrast, rely on the exploitation of the dynamic model of the manipulator for which the reference inputs are planned. Among model-based methods, it is common to cast motion design problem as an optimal control one, thus allowing to employ the results of an extensive and mature literature [4]. The motion design methods rooted in optimal control can further be split between direct and indirect methods.

Direct methods use a proper discretized description of the kinematic quantities of the multibody system to translate an optimal control problem into a parameter optimization one [5]. The resulting finite-dimensional problem is therefore solvable using one of the many parametric optimization algorithms.

Indirect methods are instead based on calculus of variations, which is used to set-up and solve the motion design problem as a Two-Point Boundary Value Problem (TPBVP). Successful applications of this methods are found, among others, for flexible link robots [6], flexible joint robots [7], mobile robots [8], lumped spring-mass systems with nonlinear springs [9], and for cable-driven robots [10], [11], [12] . Indirect control methods are generally chosen for their accuracy [4], however their field of application is often limited to small scale problems by the difficulty of finding a numerical solution of the problem under investigation. This limitation is commonly referred to as the 'curse of dimensionality' [13]. Additionally, most of the indirect methods proposed so far have limited robustness to mismatches between the description of the plant used for planning and the actual system dynamics. This problem has been extensively dealt with in the field of closed-loop control, while it has received less attention in the field of motion planning. Among the few works that, to the best of authors' knowledge, focus explicitly on robust motion design, one is [14], in which the optimization problem is mode robust by introducing in the cost function a Gaussian cumulative noise term. Another relevant work is [15], in which the authors take into account the effects of a varying payload on the motion design through the modification of bounds on the joint torques.

A different approach to model-based motion planning is the well-known input shaping technique. Input shaping motion planning techniques work by convolving a reference signal, either a position reference or a force reference, with a baseline of impulses to ensure reduced residual vibrations. The popularity of shaping filters is motivated by their very straightforward implementation and for being suitable to a wide range of applications, such as in the movement of suspended loads [16], [17]. Shaping techniques can be, however, unsuitable in some applications, such as the ones in which the time delay required by the shaping action is close 
to the specified motion time dictated by the application. The issue of robustness of input shaping has been also widely discussed, leading to several robust versions such as the ZVD shaper [18]. Increasing robustness however exacerbates the aforementioned problem of the delayed response.

This work proposes an indirect approach to robust motion planning in underactuated multibody systems. By exploiting the mathematical frame proposed in [19] for inverse dynamics, and then extended in [20], the method computes the optimal reference trajectory for rest-to-rest motion of an underactuated multibody system to accomplish several design tasks. First, it should ensure reduced transient load oscillation, both in term of peak and average value, even in the execution of fast motions. Secondly, reduced control effort is required, in term of reduced amplitude and harmonic content of the required accelerations for the actuated coordinates. Finally, robustness with respect to an uncertain critical parameter should be granted. The numerical results obtained provide a clear evidence of the improved capability of the proposed method over similar input shaping methods when dealing with fast motion applications.

\section{System model formulation}

Let $n$ be the number of degrees of freedom of the system under investigation and given a proper choice of a vector of $n$ independent coordinates $\mathbf{q}$, a set of $n$ nonlinear ordinary differential equations is used to describe its dynamics:

$$
\mathbf{M}(\mathbf{q}) \ddot{\mathbf{q}}(t)=\mathbf{K}(\mathbf{q})+\mathbf{G}(\mathbf{q}, \dot{\mathbf{q}})+\mathbf{B}(\mathbf{q}) \mathbf{F}(t)
$$

$\mathbf{M} \in \mathfrak{R}^{n \times n}$ is the mass matrix, $\mathbf{K} \in \mathfrak{R}^{n}$ is the vector of position-dependent forces while vector $\mathbf{G} \in \mathfrak{R}^{n}$ collects gyroscopic and centrifugal forces as well as the damping forces. The vector of the external actuation and control forces $\mathbf{F} \in \mathfrak{R}^{m}$ is input into the system dynamics through the force distribution matrix $\mathbf{B} \in \mathfrak{R}^{n \times m}$. Since $m<n$, the system is underactuated. The dynamic model in (1) can be rewritten by partitioning $\mathbf{q}$ into the vector of $m$ actuated generalized coordinates, and the vector $\mathbf{q}_{u}$ of the $n-m$ unuactuated ones [21]:

$$
\begin{aligned}
{\left[\begin{array}{ll}
\mathbf{M}_{a a} & \mathbf{M}_{a u} \\
\mathbf{M}_{a u}^{T} & \mathbf{M}_{u u}
\end{array}\right] } & {\left[\begin{array}{c}
\ddot{\mathbf{q}}_{a} \\
\ddot{\mathbf{q}}_{u}
\end{array}\right]=\left[\begin{array}{l}
\mathbf{K}_{a}(\mathbf{q}) \\
\mathbf{K}_{u}(\mathbf{q})
\end{array}\right] } \\
& +\left[\begin{array}{c}
\mathbf{G}_{a}(\mathbf{q}, \dot{\mathbf{q}}) \\
\mathbf{G}_{u}(\mathbf{q}, \dot{\mathbf{q}})
\end{array}\right]+\left[\begin{array}{c}
\mathbf{B}_{a} \\
0
\end{array}\right] \mathbf{F}
\end{aligned}
$$

This reformulation highlights that the motion of the unactuated coordinates is excited by the motion of the actuated ones, while is not directly affected by the external forces $\mathbf{F}$ :

$$
\ddot{\mathbf{q}}_{u}=\mathbf{M}_{u u}^{-1}\left(\mathbf{K}_{u}+\mathbf{G}_{u}\right)-\mathbf{M}_{u u}^{-1} \mathbf{M}_{a u}^{T} \ddot{\mathbf{q}}_{a}
$$

As far as the motion of the actuated coordinates is concerned, it is excited and controlled by the $m$ actuation forces collected in $\mathbf{F}$, and can be perturbed by the motion of the unactuated coordinates. However, the use of effective controllers in the actuators, that ensure high bandwidth and effective disturbance rejection (such as high gain controllers, feedforward or load observer schemes), allows the actuators to get rid of the elastic dynamics of the unactuated degrees of freedom. A similar result is obtained in the case of small inertia ratios, i.e. whenever the reflected load inertia is smaller than those of the actuators. Hence, the motion the actuated coordinates can be interpreted as the external and independent input for the dynamics of the unactuated ones. One one hand, it can excite the oscillating dynamics of the unactuated subsystem; on the other one, a proper design of such a motion can lead to reduced undesired vibrations. Under these hypotheses, the problem of optimal motion planning for load vibration control can just rely on the subsystem model in (3) in lieu of the full system model (1).

A simplified model of the actuators dynamics is instead included in the model adopted for motion planning, to account for their finite bandwidths that do not allow tracking high frequency components of the reference trajectory. The actual acceleration of the actuated coordinates is therefore written as a function of the reference acceleration $\ddot{\mathbf{q}}_{a}^{r e f}(t)$ :

$$
\ddot{\mathbf{q}}_{a}(t)=\mathbf{h}\left(\ddot{\mathbf{q}}_{a}^{r e f}(t)\right)
$$

The overall dynamic model adopted for the trajectory synthesis is therefore the following one, where the exogenous input is $\ddot{\mathbf{q}}_{a}^{r e f}(t)$ :

$$
\begin{aligned}
{\left[\begin{array}{c}
\ddot{\mathbf{q}}_{u} \\
\ddot{\mathbf{q}}_{a}
\end{array}\right]=} & {\left[\begin{array}{l}
\mathbf{M}_{u u}^{-1}\left(\mathbf{K}_{u}(\mathbf{q})+\mathbf{G}_{u}(\mathbf{q}, \dot{\mathbf{q}})\right) \\
0
\end{array}\right] } \\
& +\left[\begin{array}{c}
-\mathbf{M}_{u u}^{-1} \mathbf{M}_{a u}^{T} \\
\mathbf{I}
\end{array}\right] \mathbf{h}\left(\ddot{\mathbf{q}}_{a}\right)
\end{aligned}
$$

The re-formulation proposed in (5) is suitable to compute, as the output of the design algorithm, the reference profile $\mathbf{q}_{\text {ref }}$ for commanding each actuator. This approach has three paramount advantages over the use of the full dynamic model. First of all, 
it reduces the number of uncertain parameters and does not need precise models of friction forces on the actuated coordinates, which are instead required if optimal motion planning is aimed at computing the optimal control force (i.e. an inverse dynamics problem) to ensure no steady-state errors. The computation of the control forces to be exerted by the actuators is here demanded to the axis control of each actuator (e.g. to its native controller). The second advantage is that smaller models are more reliable under a computational point of view, being usually better conditioned. Finally, the optimal reference for the actuated coordinates can be used as the position reference of the actuators even if the native position and speed controllers are adopted. Indeed, these 'closed' proprietary control schemes cannot be usually modified in industrial mechatronic systems and they are often designed to accept a position reference signal as the only exogenous input.

A simple and effective way to model $\mathbf{h}$ in (5) is to approximate it through its dominant poles. This is one of the few, if not the only, available options when the manufacturers' data sheet provide little or no information on the actuation and control implementation. If the closed-loop system is nearly critically damped, the closed-loop bandwidth value can be incorporated just through the time constant $\tau$ of the first-order linear model:

$$
\dddot{\mathbf{q}}_{a}(t)=-\frac{1}{\tau} \ddot{\mathbf{q}}_{a}(t)+\frac{1}{\tau} \ddot{\mathbf{q}}_{a}^{r e f}(t)
$$

In practice, $\tau$ behaves also as a smoothing parameter that can be also wisely tuned to reduce the harmonic content of the reference trajectory. The inclusion of (6) into (5) requires augmenting the state vector with $\ddot{\mathbf{q}}_{a}$ as follows:

$$
\begin{aligned}
{\left[\begin{array}{c}
\ddot{\mathbf{q}}_{u} \\
\ddot{\mathbf{q}}_{a}
\end{array}\right]=} & {\left[\begin{array}{c}
\mathbf{M}_{u u}^{-1}\left(\mathbf{K}_{u}(\mathbf{q})+\mathbf{G}_{u}\left(\underset{\mathbf{q}}{\ddot{\mathbf{q}}_{a}}\right)\right)-\mathbf{M}_{u u}^{-1} \mathbf{A}_{a u}^{T} \ddot{\mathbf{q}}_{a} \\
-\frac{\ddot{q}_{a}}{\tau}
\end{array}\right] } \\
& +\left[\begin{array}{c}
0 \\
\frac{1}{\tau}
\end{array}\right] \ddot{\mathbf{q}}_{a}^{\text {ref }}
\end{aligned}
$$

This model formulation can be finally cast to fit the classical form of a set of first-order ordinary differential equations, as required for solving the trajectory optimization problem, with $\mathbf{u}$ the control vector and $\mathbf{x}$ the state vector:

$$
\mathbf{u}=\ddot{\mathbf{q}}_{a}^{r e f} ; \quad \mathbf{x}=\left[\dot{\mathbf{q}}_{u}, \ddot{\mathbf{q}}_{a}, \dot{\mathbf{q}}_{a}, \mathbf{q}_{u}, \mathbf{q}_{a}\right]^{T} ; \quad \dot{\mathbf{x}}=\mathbf{f}(\mathbf{x}, t, \mathbf{u})
$$

\section{Variational formulation of robust trajectory planning}

The formulation of the proposed robust trajectory problem applies to point-to-point motion, for which the initial and the final configurations of the system are the obvious choice as the 'left' and 'right' boundary conditions [4]. Since the state trajectory is free between the two boundary points, and hence an infinite number of trajectories connecting them can be defined, the proposed approach aims at finding the one that minimizes a suitable cost function $J$ :

$$
J=\int_{t_{0}}^{t_{f}} g(\mathbf{x}, t, u) d t
$$

The scalar function $g$ can be chosen arbitrarily to fulfill the required goals of the trajectory design. By combining the cost function with the two boundary conditions on $\mathbf{x}(t)$ and treating the system model as a constraint, the following optimization problem is defined:

$$
\left\{\begin{array}{l}
\min J(\mathbf{x}(t), t, \mathbf{u})=\min \int_{t_{0}}^{t_{f}} g(\mathbf{x}, t, \mathbf{u}) d t \\
\text { subject to }: \\
\mathbf{x}\left(t_{0}\right)=\mathbf{x}_{0} ; \quad \mathbf{x}\left(t_{f}\right)=\mathbf{x}_{f} ; \\
\dot{\mathbf{x}}(t)=\mathbf{f}(\mathbf{x}, t, \mathbf{u}) ;
\end{array}\right.
$$

The most common way to solve such problem is to use the calculus of variations, such as the Pontryagin's Minimum Principle (PMP) [22]. To improve the optimization problem in (10) with robustness specifications, it can be augmented with some sensitivity functions, as suggested in the previous work of one of the Authors [19].

Let us assume that the dynamic model of the system is affected by the uncertain scalar parameter $\mu$, and therefore eq. (8) can be written as:

$$
\dot{\mathbf{x}}(t)=\mathbf{f}(\mathbf{x}, t, \mathbf{u}, \mu)
$$

If $\mathbf{f}$ is continuous in $(\mathbf{x}, t, \mu)$ and is continuously differentiable with respect to $\mathbf{x}$ and $\mu$ for any value of $(\mathbf{x}, t, \mu)$ in the interval $\left[t_{0}, t\right]$, then the system response $\mathbf{x}(t, \mu)$ can be evaluated as:

$$
\mathbf{x}(t, \mu)=\mathbf{x}\left(t_{0}\right)+\int_{t_{0}}^{t} \mathbf{f}(s, \mathbf{x}(s), \mu) d s
$$


Since the latter is differentiable, the partial derivative of the state with reference to the uncertain parameter $\mu$, denoted as $\mathbf{S}(t)$, can be computed as:

$$
\begin{aligned}
\mathbf{S}(t)=\frac{\partial \mathbf{x}(t)}{\partial \mu}=\int_{t_{0}}^{t} & {\left[\frac{\partial \mathbf{f}(s, \mathbf{x}(s, \mu), \mu)}{\partial \mathbf{x}} \frac{\partial \mathbf{x}(s, \mu)}{\partial \mu}\right.} \\
+ & \left.\frac{\partial \mathbf{f}(s, \mathbf{x}(s, \mu), \mu)}{\partial \mu}\right] d s
\end{aligned}
$$

$\mathbf{S}(t)$ describes how the system response is affected by the deviation of $\mu$ from its nominal value $\mu_{0}$. One way to obtain small sensitivity is to enforce its amplitude at the "critical" time instants of the motion, such as the initial and final ones $t_{0}$ and $t_{f}$. To do so, the motion design problem (10) should be re-cast by augmenting the dynamic model and the state vector:

$$
\begin{aligned}
\dot{\mathbf{x}}^{r}(t)=\mathbf{f}^{r}(\mathbf{x}, \mathbf{S}, t, \mathbf{u}, \mu) & =\left[\begin{array}{c}
\mathbf{f}(\mathbf{x}, t, \mathbf{u}, \mu) \\
\frac{\partial \mathbf{f}(\mathbf{x}, t, \mathbf{u}, \mu)}{\partial \mu}
\end{array}\right]_{\mu=\mu_{0}} \\
\mathbf{x}^{r}(t) & :=\left[\begin{array}{c}
\mathbf{x}(t) \\
\mathbf{S}(t)
\end{array}\right]
\end{aligned}
$$

The 'robustification' (or 'desensitization') procedure through the inclusion of sensitivity functions therefore leads to the definition of the robust optimization problem of eq. (16):

$$
\left\{\begin{array}{l}
\min J^{r}\left(\mathbf{x}^{r}, t, \mathbf{u}\right)=\min \int_{t_{0}}^{t_{f}} g^{r}\left(\mathbf{x}^{r}, t, \mathbf{u}, \mu\right) d t \\
\text { subject to }: \\
\mathbf{x}\left(t_{0}\right)=\mathbf{x}_{0} ; \quad \mathbf{x}\left(t_{f}\right)=\mathbf{x}_{f} \\
\mathbf{S}\left(t_{0}\right)=0 ; \quad \mathbf{S}\left(t_{f}\right)=0 \\
\dot{\mathbf{x}}(t)=\mathbf{f}(\mathbf{x}(t), t, \mathbf{u}, \mu) ; \quad \dot{\mathbf{S}}(t)=\frac{\partial \mathbf{f}(\mathbf{x}(t), t, \mathbf{u})}{\partial \mu}
\end{array}\right.
$$

The optimization problem in eq. (16) can be solved using the Pontryagin's Minimum Principle (PMP), which exploits the Hamiltonian of the system:

$$
\mathscr{H}=g^{r}+\boldsymbol{\lambda}^{T} \mathbf{f}^{r}\left(\mathbf{x}^{r}, t, \mathbf{u}\right)
$$

The Hamiltonian function includes the vector of Lagrangian multipliers $\boldsymbol{\lambda} \in R^{N}$ to constrain the solution to the system dynamics. The three conditions imposed by the PMP are then:

$$
\dot{\mathbf{x}}=\frac{\partial \mathscr{H}}{\partial \boldsymbol{\lambda}} ; \quad \dot{\lambda}=-\frac{\partial \mathscr{H}}{\partial \mathbf{x}} ; \quad \frac{\partial \mathscr{H}}{\partial \mathbf{u}}=0
$$

This problem is usually solved numerically through collocation [23] or shooting techniques [24]. Analytic solutions are usually practical only for simple problems.

\section{Model of the application example}

The underactuated multibody system under investigation consists of two masses $\left(m_{1}\right.$ and $\left.m_{2}\right)$ suspended to a translating cart through two ropes, whose lengths are $L_{1}$ and $L_{2}$. The following values have been assumed: $m_{1}=0.192 \mathrm{~kg}, m_{2}=0.201 \mathrm{~kg}$, $L_{1}=0.470 \mathrm{~m}, L_{2}=0.391 \mathrm{~m}, \tau=0.1 \mathrm{~s}$. The motion to be performed is, for all tests, a rest-to-rest displacement with $0.3 \mathrm{~m}$ amplitude to be completed in $3 \mathrm{~s}$. According to Fig. 1, the cart position is denoted $y_{\text {cart }}$, while the angular displacement of the two masses are defined through angles $\theta_{1}$ and $\theta_{2}$. Using $u(t)=\ddot{y}_{\text {cart }}^{\text {ref }}$ as the input of the dynamic model of the subsystem representing the load, the dynamic model can be specified, according to the notation of (7), by the following matrices:

$$
\begin{gathered}
\mathbf{B}_{a}=1 ; \quad \mathbf{K}_{a}=0 ; \quad \mathbf{G}_{a}=0 ; \\
\mathbf{M}_{a u}=\left[\begin{array}{cc}
L_{1}\left(m_{1}+m_{2}\left(1+L_{2} \cos \left(\theta_{2}\right)\right)\right) & m_{2} L_{2} \cos \left(\theta_{2}\right) \\
L_{1} \cos \left(\theta_{2}\right)+L_{2} & L_{2}
\end{array}\right] \\
\mathbf{K}_{u}=\left[\begin{array}{c}
-g\left(m_{1}+m_{2}\right) \sin \left(\theta_{1}\right)+\ddot{y}_{c a r t}\left(m_{1}+m_{2}\right) \cos \left(\theta_{1}\right) \\
-g \sin \left(\theta_{1}+\theta_{2}\right)+\ddot{y}_{c a r t} \cos \left(\theta_{1}+\theta_{2}\right)
\end{array}\right]
\end{gathered}
$$






Figure 1: Kinematic model of the double-pendulum crane

$$
\mathbf{G}_{u}=\left[\begin{array}{c}
m_{2} L_{2}\left(\dot{\theta}_{1}^{2}+\dot{\theta}_{2}^{2}\right) \sin \left(\theta_{2}\right) \\
-L_{1}^{2} \dot{\theta}_{1}^{2} \sin \left(\theta_{2}\right)
\end{array}\right]
$$

The first-order model required for trajectory planning is based on the following state vector:

$$
\mathbf{x}(t)=\left[\ddot{y}_{\text {cart }}, \dot{\theta}_{1}, \dot{\theta}_{2}, \dot{y}_{\text {cart }}, \theta_{1}, \theta_{2}, y_{\text {cart }}\right]^{T}
$$

The 'robustification' is made with respect to $L_{1}$, which significantly affects both natural frequencies $\omega_{1}$ and $\omega_{2}$ [16]. The augmented state vector is therefore:

$$
\mathbf{x}^{r}(t)=\left[\ddot{y}_{c a r t}, \dot{\theta}_{1}, \dot{\theta}_{2}, \dot{y}_{c a r t}, \theta_{1}, \theta_{2}, y_{c a r t}, \dot{s}_{1}, \dot{s}_{2}, s_{1}, s_{2}\right]^{T}
$$

which includes the sensitivity functions:

$$
\begin{array}{ll}
s_{1}(t)=\frac{\partial \theta_{1}(t)}{\partial L_{1}} ; & s_{2}(t)=\frac{\partial \theta_{2}(t)}{\partial L_{1}} ; \\
\dot{s}_{1}(t)=\frac{\partial \dot{\theta}_{1}(t)}{\partial L_{1}} ; & \dot{s}_{2}(t)=\frac{\partial \dot{\theta}_{2}(t)}{\partial L_{1}} ;
\end{array}
$$

The sensitivity functions are evaluated analytically using (14) and (15). It should also be pointed out that the sensitivity function $\partial y_{\text {cart }} / \partial L_{1}$ and its time derivatives are not included in the model since they are always zero, being $y_{\text {cart }}(t)$ independent form $L_{1}$ in the model of the subsystem assumed here.

\section{Load sway reduction: numerical results and comparison with input shapers}

The performance evaluation of the proposed method is conducted through numerical simulation and the comparison with two benchmark input shaping methods: the Negative Zero Vibration (NZV) shaper [25], and the Zero Vibration Derivative (ZVD) shaper [26]. The goal of the motion planning is to design a motion profile for rest-to-rest motion with negligible residual and transient oscillations, as well as with enhanced robustness. The requirement of zero residual vibrations for the nominal case is obtained by imposing, in the optimization problem, null values for $\theta_{1}, \theta_{2}$ and for their time derivatives at final time $t_{f}$. As for the transient behaviour, it is required to reduce both the 'average' and the peak values of the relative displacement between the cart and the end-point mass, while ensuring reasonably small accelerations. To satisfy these requirements, function $g$ has been defined as follows:

$$
g=\frac{1}{2} u^{2}+\beta \exp \left(\left(\gamma\left(L_{1} \sin \left(\theta_{1}\right)+L_{2} \sin \left(\theta_{1}+\theta_{2}\right)\right)\right)^{2}\right)
$$

Such a definition of $g$ trades off between the reduction of the acceleration reference signal $u(t)$, i.e. the control effort, and the load oscillation, which is weighed by the positive scalar weight $\beta$ and by the scaling factor $\gamma$. The use of the exponential emphasizes the peaks of the oscillation in the cost function, thus keeping them small. In practice it roughly approximates a kind of minmax optimization problem within the frame of the classical optimal control, thus allowing for a straightforward numerical solution. The possibility of defining different objective functions to accomplish various secondary goals, besides the usual requirement of zero residual vibration, is an important advantage of the variational approach over input shaping. Indeed, input shaping motion planning techniques work by convolving an arbitrary reference signal with a sequence of impulses whose number, amplitudes and times of application depend on the natural frequency and damping of the vibrational modes to control, and on the desired robustness. Other control objectives, such as reduction of the transient oscillations and of the control effort, should be obtained by a clever selection of the original reference signal to be convolved with the shaper. The problem of reducing transient oscillations and control effort is exacerbated in three cases: if the desired motion time is comparable with the period of natural oscillation, 


\begin{tabular}{c||cccc}
\multicolumn{6}{c}{ Table 1: Performance measurements } \\
\hline Test & $\ddot{y}_{\max }$ & $\ddot{y}_{R M S}$ & $\dddot{y}_{\max }$ & $\dddot{y}_{R M S}$ \\
& {$\left[\mathrm{~m} / \mathrm{s}^{2}\right]$} & {$\left[\mathrm{m} / \mathrm{s}^{2}\right]$} & {$\left[\mathrm{m} / \mathrm{s}^{3}\right]$} & {$\left[\mathrm{m} / \mathrm{s}^{3}\right]$} \\
\hline \hline $\mathrm{NZV}$ & 0.2141 & 0.1299 & 1.6982 & 0.7381 \\
ZVD & 0.7067 & 0.3727 & 20.5333 & 6.0468 \\
TPBVP, $\beta=0$ & 0.3337 & 0.1840 & 3.7308 & 10.0657 \\
TPBVP, $\beta=20$ & 0.5571 & 0.2283 & 19.2325 & 3.6195 \\
TPBVP, $\beta=50$ & 0.7030 & 0.2835 & 25.5660 & 4.2066 \\
\hline
\end{tabular}

if the desired robustness increases, and in the presence of more vibrational modes to control. Indeed, the convolution of the unshaped reference with the shaper impulses introduces delays that are higher as the natural frequency decreases. The number of such impulses is higher for robust shapers and for multi-mode shapers. The latter, indeed, are obtained by cascading two or more shaping filters. Hence, the only way to retain the original motion time is to pre-compensate the delay by reducing the time duration of the reference signal prior to shaping. This feature can be a limiting factor when the overall time delay is comparable with the required motion time, since the unshaped motion profiles have very high accelerations that cause high transient oscillations.

In the proposed test case, both the shapers have been fed with a displacement reference profile described by a fifth-degree polynomial function, to ensure continuous accelerations, whose duration is set to compensate for the delay introduced by the shapers. Since $\omega_{1}=3.640 \mathrm{rad} / \mathrm{s}$ and $\omega_{2}=9.017 \mathrm{rad} / \mathrm{s}$ in the nominal model (null damping is assumed), the NZV shaper introduces a delay equal to $0.808 \mathrm{~s}$, while the ZVD shaper delay is equal to $1.789 \mathrm{~s}$. Hence, the total times for the unshaped motions should be set to $2.192 \mathrm{~s}$ and $1.211 \mathrm{~s}$, respectively. Two sample values of $\beta$, i.e. $\beta=0$ and $\beta=50$, have been chosen to solve the TPVBP problem, to show how it affects the amplitude of the transient load oscillation. The scaling factor $\gamma$ is set to 50 for all tests, which has proved to produce a proper weighting factor for the sway angles $\theta_{1}$ and $\theta_{2}$. Moreover, it has been observed that using two variable weighting factor do not provide significant performance improvements. All the reference speed and acceleration profiles for cart are shown in Fig. 2. Figure 2 and 3 show that, for the test-case under consideration, the ZVD shaper requires higher actuator effort than the other two methods, given that it requires the highest RMS cart acceleration $\left(0.3727 \mathrm{~m} / \mathrm{s}^{2}\right)$. The lowest actuator effort is required by the NZV shaper, which requires a RMS acceleration as low as $0.1299 \mathrm{~m} / \mathrm{s}^{2}$. However, it does not share the robustness sported by the two other methods, which can be judged by measuring the residual vibrations in 5 . It should be also pointed out that jerk continuity is obtained only by the proposed method: obtaining jerk continuity with input shaping techniques requires polynomials with higher degree, and hence even higher accelerations. The load oscillations for the three motion profiles are reported in Fig. 4 and 5. Figure 4 refers to the nominal conditions: the NZV shaper, the ZVD shaper and the variational solution with $\beta=0$ have similar transient load oscillation and null residual ones. In contrast, a sensible peak load oscillation is obtained by setting $\beta=50$, as expected, at the cost of a small increase of the cart accelerations. Figure 5 simulates a mismatch between the actual system and the model adopted for the synthesis, in which the length of the first cable, $L_{1}$, is increased by $20 \%$. In this case, the residual load oscillation is rather small for all three robust methods, while the only non-robust one, i.e. the NZV shaper, produces a residual peak load oscillation that is roughly one third of the transient one. The proposed method with $\beta=0$ and the ZVD shaper lead to very similar results, while setting $\beta=50$ can reduce even further the amplitude of residual vibrations. Tables 1 and 2 summarize all these results.
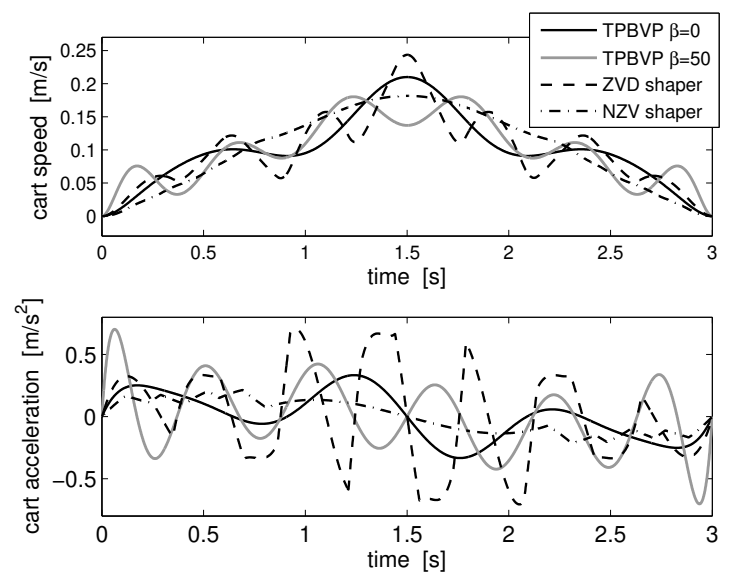

Figure 2: Planned cart speed and acceleration: proposed method with $\beta=0$ and $\beta=50$, NZV and ZVD shaper 

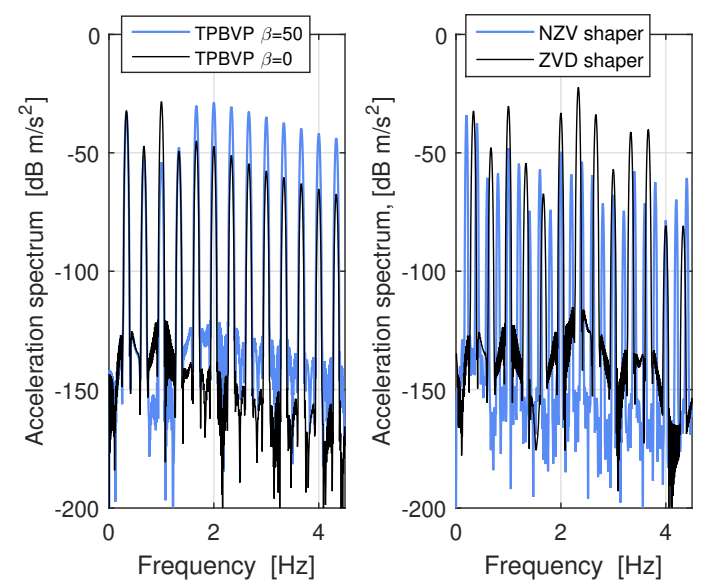

Figure 3: Spectrum of planned cart acceleration: proposed method with $\beta=0$ and $\beta=50$, NZV and ZVD shaper

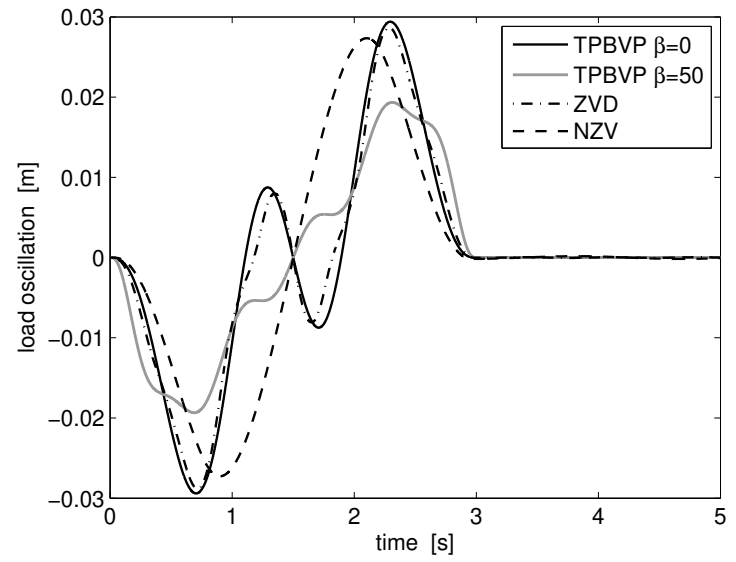

Figure 4: Load oscillation with unperturbed plant

Table 2: Performance measurements: peak ans RMS load oscillation, measured in $[\mathrm{mm}]$

\begin{tabular}{c||cccc}
\hline Test & $\begin{array}{c}\text { transient } \\
\text { peak }\end{array}$ & $\begin{array}{c}\text { transient } \\
\text { RMS }\end{array}$ & $\begin{array}{c}\text { residual } \\
\text { peak }\end{array}$ & $\begin{array}{c}\text { residual } \\
\text { peak, } \Delta L_{1}=20 \%\end{array}$ \\
\hline \hline NZV & 27.347 & 17.037 & 0.169 & 8.850 \\
ZVD & 28.807 & 14.917 & 0.042 & 1.622 \\
TPBVP $\beta=0$ & 29.404 & 15.817 & 0.000 & 1.774 \\
TPBVP $\beta=20$ & 21.984 & 12.943 & 0.001 & 1.470 \\
TPBVP $\beta=50$ & 19.646 & 12.374 & 0.001 & 1.465 \\
\hline
\end{tabular}

\section{Conclusion}

In this work a method for the robust design of motion profiles for underactuated multibody systems has been presented. The method is based on the definition of a robust optimization problem constrained to the plant dynamics, which is set-up with the aid of parametric sensitivity functions and by exploiting the Pontryagin's Minimum Principle. The performances are assessed by comparison with the application of NZV and ZVD shapers, showing that, for the case under consideration, the proposed method has similar robustness properties of the widely adopted ZVD shaper, while requiring lower actuator effort and bandwidth. Additionally, a suitable definition of the cost function adopted for the synthesis of the motion profile, allows significantly reducing the peak transient oscillation at the cost of a minor increase of the control effort.

\section{References}

[1] A. Gasparetto, P. Boscariol, A. Lanzutti, R. Vidoni. Trajectory planning in robotics. Mathematics in Computer Science, 1-11, 2012. 




Figure 5: Load oscillation with perturbed plant $\left(\Delta L_{1}=+20 \%\right)$

[2] P. Barre, R. Bearee, P. Borne, E. Dumetz. Influence of a jerk controlled movement law on the vibratory behaviour of high-dynamics systems. Journal of Intelligent \& Robotic Systems, 42(3):275-293, 2005.

[3] A. Gasparetto, V. Zanotto. A technique for time-jerk optimal planning of robot trajectories. Robotics and ComputerIntegrated Manufacturing, 24(3):415-426, 2008.

[4] D. Kirk. Optimal control theory: an introduction. Dover Pubns, 2004.

[5] J. T. Betts. Survey of numerical methods for trajectory optimization. Journal of guidance, control, and dynamics, 21(2):193207, 1998.

[6] O. Dahl. Path constrained motion optimization for rigid and flexible joint robots. In International Conference on Robotics and Automation, volume 2, 223-229. IEEE, 1993.

[7] P. Boscariol, A. Gasparetto. Model-based trajectory planning for flexible-link mechanisms with bounded jerk. Robotics and Computer-Integrated Manufacturing, 29(4):90-99, 2013.

[8] D. Balkcom, M. Mason. Time optimal trajectories for bounded velocity differential drive vehicles. The International Journal of Robotics Research, 21(3):199-217, 2002.

[9] C. M. Pappalardo, D. Guida. Adjoint-based optimization procedure for active vibration control of nonlinear mechanical systems. Journal of Dynamic Systems, Measurement, and Control, 139(8):081010, 2017.

[10] M. Bamdad. Time-energy optimal trajectory planning of cable-suspended manipulators. In Cable-Driven Parallel Robots, 41-51. Springer, 2013.

[11] A. Idà, Edoardo andBerti, T. Bruckmann, M. Carricato. Rest-to-rest trajectory planning for planar underactuated cabledriven parallel robots. Cable-Driven Parallel Robots, Mechanisms and Machine Science, vol 53.:207-218, 2018.

[12] E. Idà, T. Bruckmann, M. Carricato. Robust point-to-point trajectory planning for nonlinear underactuated systems: Theory and experimental assessment. IEEE Transactions on Robotics, 35:1338-1351, 2019.

[13] R. Bellman, et al. The theory of dynamic programming. Bulletin of the American Mathematical Society, 60(6):503-515, 1954.

[14] D. Gallardo, O. Colomina, F. Flórez, R. Rizo. A genetic algorithm for robust motion planning. In International Conference on Industrial, Engineering and Other Applications of Applied Intelligent Systems, 115-121. Springer, 1998.

[15] K. Shin, N. McKay. Robust trajectory planning for robotic manipulators under payload uncertainties. IEEE transactions on Automatic Control, 32(12):1044-1054, 1987.

[16] W. Singhose, D. Kim, M. Kenison. Input shaping control of double-pendulum bridge crane oscillations. Journal of Dynamic Systems, Measurement, and Control, 130(3):034504, 2008.

[17] T. Singh. Optimal reference shaping for dynamical systems: theory and applications. CRC Press, 2009.

[18] N. C. Singer, W. P. Seering, et al. Preshaping command inputs to reduce system vibration. Massachusetts Institute of Technology, Artificial Intelligence Laboratory, 1988. 
[19] P. Boscariol, A. Gasparetto. Robust model-based trajectory planning for nonlinear systems. Journal of Vibration and Control, 22(18):3904-3915, 2016.

[20] P. Boscariol, D. Richiedei. Rest-to-rest trajectory planning for underactuated cable-driven parallel robots. Robotics and Computer-Integrated Manufacturing, 50:256-265, 2018.

[21] R. Belotti, D. Richiedei, I. Tamellin, A. Trevisani. Response optimization of underactuated vibration generators through dynamic structural modification and shaping of the excitation forces. The International Journal of Advanced Manufacturing Technology, 112:505-524, 2021.

[22] L. Pontriagin, R. Gamkrelidze. The mathematical theory of optimal processes, volume 4. CRC, 1986.

[23] L. Shampine, I. Gladwell, S. Thompson. Solving ODEs with MATLAB. Cambridge Univ Pr, 2003.

[24] R. W. Holsapple. A modified simple shooting method for solving two-point boundary value problems. Ph.D. thesis, Texas Tech University, 2003.

[25] W. Singhose, W. Seering, N. C. Singer. Time-optimal negative input shapers. Journal of Dynamic Systems, Measurement, and Control, 119(2):198-205, 1997.

[26] W. Singhose. Command shaping for flexible systems: A review of the first 50 years. International Journal of Precision Engineering and Manufacturing, 10(4):153-168, 2009. 